\title{
Reconstruction of Subcortical Brain Activity by Spatially Filtered MEG During Epileptic Seizures
}

\author{
H. Kober, O. Ganslandt, Ch. Nimsky, M. Buchfelder, and R. Fahlbusch \\ Neurocenter, Dept. of Neurosurgery, University Erlangen-Nuremberg, Germany \\ kober@nch.imed.uni-erlangen.de
}

\begin{abstract}
We analyzed the spontaneous brain activity of a brain tumor patient during an epileptic seizure. The current density reconstruction by spatial filtering (CLSF) revealed pathologic, neuronal activity originating from the hippocampus ipsilateral to the tumor which was confirmed by intraoperative electrocorticography. This study shows that subcortical brain activity can be localized noninvasively using MEG. Further MEG studies combined with depth electrode recordings will have to show the validity of our results and might help to improve our understanding of the pathophysiology of epileptogenesis.
\end{abstract}

Introduction Cortical interictal and subclinical ictal epileptic brain activity can be localized by Magnetoencephalography (MEG) [1]. Depth electrode recordings and lesion studies in epilepsy patients have shown that deep temporomesial structures like the hippocampus (HC) are important for the pathophysiology of epileptogenesis. However, depth electrode recordings show that with conventional dipole localization approaches activity originating from deep brain structures is likely to escape detection [2]. To noninvasively elucidate time course and location of these sources during epileptiform discharges we used a localization approach based on spatial filtering taking into account the spatio-temporal information of the measured MEG signal.

Methods We recorded bilateral spontaneous MEG activity in a 41 year old female patient with a brain tumor and focal epilepsy with a $2 \times 37$ channel biomagnetic system Magnes II (4-D Neuroimaging, San Diego, USA). Time course and location of ictal epileptiform discharges were investigated using a current density localization approach by spatial filtering (CLSF) [3]. The magnetic flux density $B_{i}$ measured with a MEG sensor with lead field $G_{i}$ which is generated by a current density distribution $J$ can be calculated by $B_{i}=\int_{\Omega} \boldsymbol{J}(\boldsymbol{r}) \cdot \boldsymbol{G}(\boldsymbol{r}) d r^{3}$. The current density $J_{\theta}$ was reconstructed by a linear projection of the measured flux densities $B_{i}$ at $N$ sensor positions through a set of spatial filters $w_{i}^{\theta}$ :

$$
J_{\theta}=\sum_{i=1}^{N} B_{i} w_{i}^{\theta} .
$$

The $N$ spatial filters for a target source at a specific location and current vector indicated by $\theta$ are constructed to minimize in a least-square sense the difference between the spatial response of the virtual sensor and an ideally selective delta function:

$$
\chi^{2}=\int_{\Omega}\left[\sum_{i=1}^{N} \boldsymbol{G}_{i}(\boldsymbol{r}) w_{i}^{\theta}(\boldsymbol{r})-\boldsymbol{\delta}^{\theta}(\boldsymbol{r})\right]^{2} d r^{3} .
$$


The filter coefficients minimizing this functional are given by $\boldsymbol{w}=\boldsymbol{\Gamma}^{-1} \cdot \boldsymbol{B}$, where $\Gamma_{i j}=\int_{\Omega} \boldsymbol{G}_{i}(\boldsymbol{r}) \cdot \boldsymbol{G}_{j}(\boldsymbol{r}) d r^{3}$ is the Gram matrix and $B_{i}^{\theta}=\int_{\Omega} \boldsymbol{G}_{i}(\boldsymbol{r}) \cdot \boldsymbol{\delta}^{\theta}(\boldsymbol{r}) d r^{3}$ is the forward solution for the physical sensors due to a target dipole $\theta$.

To improve the selectivity of the spatial filters for signal inherent sources the Gram matrix is substituted by the covariance matrix of the measured MEG data. Regularisation is achieved by a truncated singular value decomposition of the data covariance matrix including only the most significant singular values for the calculation of the spatial filter coefficients. Using equation (1) either a current density distribution of instantaneous source activity can be reconstructed using a set of equally spaced filters or the time course of a source at a specific voxel for successive measurement time instances.

Results During the seizure where the patient only perceived imaginary tones (no movement) two simultaneous sources could be localized ipsilateral to the low grade glioma, one source in the $\mathrm{HC}$ and the other one in the primary auditory cortex in the superior temporal gyrus (Heschl's gyrus) adjacent to the tumor which is perfectly consistent with the clinical symptoms. The reconstructed time course of the voxels with maximum CLSF current intensity in both foci revealed that the activity in the $\mathrm{HC}$ clearly preceded the activity in Heschl's Gyrus indicating a pacemaker function of the HC during the seizure (Fig. 1). Before the surgical removal of the tumor intraoperative electrocorticography confirmed the pathologic activity in the $\mathrm{HC}$.
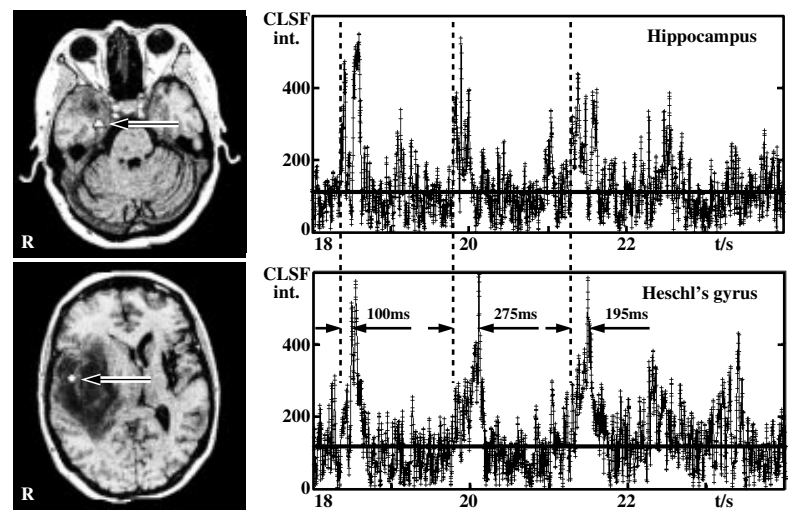

Fig. 1. Voxel with maximum CLSF intensity in axial MRI image (left) and the corresponding reconstructed time course (right) in the right hippocampus (upper row) and the right Heschl's gyrus (lower row) during an epileptic seizure with only auditory halluzinations (no movement).

\section{References}

[1] H. Stefan and C. Hummel. Handbook of Clinical Neurology, The Epilepsies. Part I, volume 72, chapter Magnetoencephalography, pages 319-336. Elsevier Science, 1999.

[2] C. Baumgartner, E. Pataraia, G. Lindinger, and L. Deecke. Neuromagnetic Recordings in Temporal Lobe Epilepsy. J Clin Neurophysiol, 17(2):177-189, 2000.

[3] S.E. Robinson and D.F. Rose. Current source image estimation by spatially filtered MEG. In M. Hooke, editor, Biomagnetism: Clinical aspects, pages 761-765. Elsevier Science, 1992. 